\title{
Controlling the Stormram 2: An MRI-compatible Robotic System for Breast Biopsy
}

\author{
Mohamed E. M. K. Abdelaziz ${ }^{1}$, Vincent Groenhuis ${ }^{1}$, Jeroen Veltman ${ }^{2}$, Françoise Siepel $^{1}$, Stefano Stramigioli $^{1}$
}

\begin{abstract}
Breast cancer is the most frequently lifethreatening diagnosed type of cancer among women. Early and accurate diagnosis by acquiring a tissue sample using biopsy techniques is essential. However, small lesions only visible by MRI are often missed in standard methods, indicating the need for a robotic-assisted biopsy system that is MRIcompatible. Existing proof-of-concepts are difficult to employ due to large sizes and/or actuation complexities. Therefore, a compact pneumatically-actuated 5 DOF MRI-compatible robot was further developed and controlled by a computerized valve manifold. Accuracy and efficiency measurements have been performed using two different PVC breast phantoms with embedded fish oil capsules (mimicking lesions) inside a $0.25 \mathrm{~T}$ MRI scanner. Preliminary results show that the end-effector was able to hit the targeted capsules, and that the position accuracy is in the range of $4.7-7.3 \mathrm{~mm}$. The developed robotic system has potential to perform MRI-guided breast biopsies accurately and improve the clinical workflow.
\end{abstract}

\section{INTRODUCTION}

Breast cancer is the most occurring invasive cancer among women, accounting for about $25 \%$ of all cases worldwide. Early diagnosis is essential for successful treatment. Because of the high sensitivity, MRI scanners can detect lesions (suspicious abnormalities) not found by mammography ( $\mathrm{x}$ ray), ultrasound or palpation. It may be necessary to perform a biopsy to extract tissue from such a lesion for accurate histological examination, requiring a needle to be inserted at the exact location, which is difficult to perform using MRI guidance. In standard procedures, the lesion is missed in approximately $10 \%$ of the cases [1], requiring repositioning of the needle leading to additional tissue damage, or possibly a false negative biopsy. Therefore, there is a request for robotic systems that can perform the biopsy more accurately and efficiently.

\section{A. State of the Art}

The MRI scanner requires that components are MRIcompatible and do not distort the magnetic field. As electromagnetic motors are ruled out, actuation of the robot is a major challenge. Alternatives have been found in pneumatics,

\footnotetext{
${ }^{1}$ Mohamed E. M. K. Abdelaziz, Vincent Groenhuis, Françoise Siepel and Stefano Stramigioli are with the Faculty of Electrical Engineering, Mathematics and Computer Science, University of Twente, Enschede, The Netherlands (e-mail: m.abdelaziz16@imperial.ac.uk, v.groenhuis@utwente.nl, f.j.siepel@utwente.nl, s.stramigiolidieee.org)

2 Jeroen Veltman is with Ziekenhuisgroep Twente, Almelo, The Netherlands (e-mail:j.veltman@zgt.nl)
}

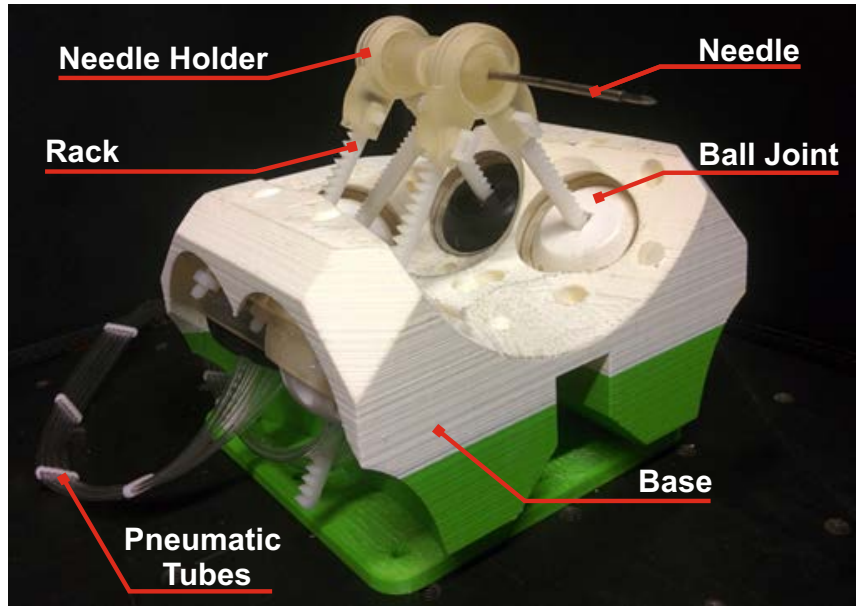

Fig. 1: The Stormram 2 robot.

hydraulics, Bowden tubes and piezoelectric actuation. Different MRI-compatible biopsy robots have been developed using (combinations of) these actuation systems. Hungr et al. developed a 5 DOF needle insertion robot driven by piezo motors coupled to the robot with Bowden tubes, combined with a pneumatic needle insertion system [2]. $\mathrm{Su}$ et al. developed different piezo/pneumatic-driven robots for prostate biopsy, of which the control electronics are placed in a shielded box inside the MRI room [3]. Yang et al. developed a piezo/pneumatic robotic system for breast biopsy [4]. Li et al. developed a transapical aortic valve replacement robot using the commercial Innomotion arm combined with a custom 3-DOF delivery module driven by pneumatic cylinders with optical feedback sensors [5]. The aforementioned robotic systems have in common that the actuation system is complex and expensive. Specialized electronics are required for driving piezoelectric actuators in a MRI environment, which also have to be located at sufficiently large distance from the scanning volume to avoid distortion of the MRI scans.

Therefore, research has been performed to develop pneumatically-driven robotic systems. Stoianovici et al. developed the six-DOF pneumatically-driven MrBot [6] for prostate intervention, but construction and control of this system is complex. In contrast, the co-authors of this paper, Groenhuis and Stramigioli, developed a low cost 7 DOF robotic system for breast biopsy driven by pneumatic linear stepper motors [7], later called the Stormram 1. It was entirely rapid prototyped by laser-cutting and 3D printing 


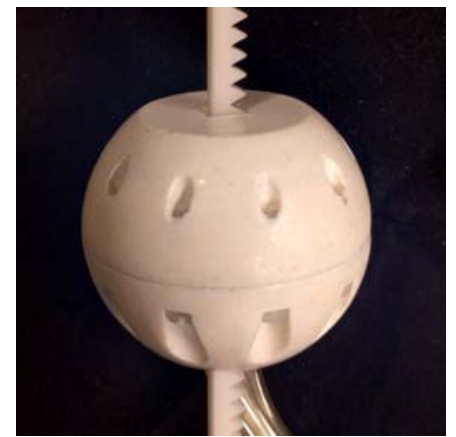

(a)

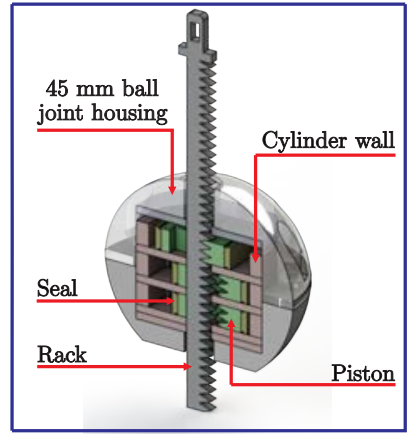

(b)
Fig. 2: (a) Assembled stepper motor, (b) Cutaway labeled view.

and control is relatively straightforward, but it is too bulky to be practical and not computer-controlled yet. The Stormram 2 design described in this paper intends to solve both issues, by miniaturization and the development of a computerized controller.

The main aim of this study is to localize the lesion in the breast phantom using MRI, and guide the robotic assisted biopsy needle of Stormram 2 towards it.

\section{Design And Implementation}

To design for maximal MRI-compatibility, a fully plastic robotic system driven by non-electric actuators was designed without using any metallic components, except for the offthe-shelf titanium needle. The pneumatic linear stepper motor principle was chosen as the actuation method because previous research has shown that this is a suitable and straightforward method for driving MRI-compatible robots. The entire robot was rapid prototyped by $3 \mathrm{D}$ printing and laser-cutting.

\section{A. Overview}

The Stormram 2 robot consists of a frame, a needle holder and interconnecting links. The frame size is $140 \mathrm{~mm} \times 140 \mathrm{~mm} \times 95 \mathrm{~mm}$ and was printed as one piece with polyactic acid (PLA) material using the Ultimaker 2 machine (Ultimaker B.V., Geldermalsen, The Netherlands). The needle holder consists of six parts, all printed in FullCure720 photopolymer material using the Objet Eden 250 machine (Stratasys Ltd., Eden Prairie, MN, USA). The needle holder is connected to the frame by means of a five-link parallel platform, which kinematic structure is inspired by MrBot [6]. Each link connects a ball joint in the frame to a joint in the needle holder. The distance between these two joints is controlled by a linear stepper motor. The off-the-shelf MRIcompatible 14-gauge titanium needle is the only metallic component of the robot, which consists of a shaft with outer diameter $2.1 \mathrm{~mm}$, and a removable inner stylet.

\section{B. Stepper motors}

Pneumatic linear stepper motors actuate the Stormram 2 robot. The design is a miniaturization of the motors used in Stormram 1 as described previously [7], such that it can

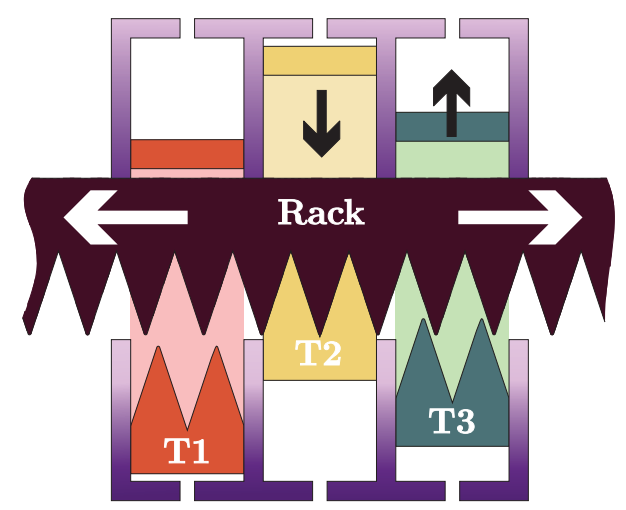

Fig. 3: Operating principle of the three pistons acting on the rack.

be embedded inside a $45 \mathrm{~mm}$ ball joint shown in Figure 2(a). It consists of two 3D printed hemispheres fixed together with nylon screws, enclosing a cavity with outer dimensions $29 \mathrm{~mm} \times 25 \mathrm{~mm} \times 26 \mathrm{~mm}$ for the motor mechanism.

1) Motor construction: The motor mechanism is entirely laser-cut from acetal and silicone rubber sheets. The internal structure can be seen in the cutaway view (Figure 2(b)). Several acetal parts (red) are stacked together to form three cavities in which toothed pistons (green) can slide. The pistons were manufactured by laser-cutting from different sides, to obtain the designed shape of the teeth. Silicone seals (yellow) are loosely attached to the piston's heads and prevent leakage of air. The bore's cross-sectional area is $12 \mathrm{~mm} \times 5 \mathrm{~mm}=60 \mathrm{~mm}^{2}$. The piston's teeth engage on a rack (white) with pitch size $3 \mathrm{~mm}$. Six pneumatic tubes with length $7 \mathrm{~m}$ and inner diameter $1.4 \mathrm{~mm}$, guide pressurized air from the valve manifold to the chambers. The nominal operating pressure is $0.4 \mathrm{MPa}$.

2) Principle of operation: Figure 3 shows the principle of operation. The three pistons move up and down individually, according to the pressurization waveform of the six chambers. Initially, the center piston (yellow) is pushed against the rack. When the right piston (green) moves up and the center one retracts, the rack is pushed one step $(1 \mathrm{~mm})$ to the right due to the wedge mechanism in the piston-teeth interaction. More steps can be performed in either direction by applying appropriate waveforms to the six chambers. This way, the relative position of the rack can be controlled in feed-forward fashion in steps of $1 \mathrm{~mm}$ using the Pulse Width Modulation (PWM) technique, which in turn moves the robot's end-effector from one waypoint to another. When the initial position is known and no steps are missed, absolute position control is possible.

3) Force calculation: The force of the piston acting on the rack can be calculated as follows. When supplied with a pressure $P=0.4 \mathrm{MPa}$, the force is:

$$
F_{p}=P \cdot A=0.4 \cdot 10^{6} \cdot 60 \cdot 10^{-6}=24 \mathrm{~N}
$$

Due to the wedge mechanism, a piston displacement of $1.5 \mathrm{~mm}$ causes a rack displacement of $1.0 \mathrm{~mm}$. Assuming ideal transfer of work, the resulting theoretical force $F_{r}$ 
exerted by the rack due to the force of one piston is $F_{r}=$ $\frac{1.5}{1} F_{p}=36 \mathrm{~N}$.

Previous measurements [8] have shown that the actual force is $15 \mathrm{~N}$ at a pressure of $0.35 \mathrm{MPa}$. At a pressure of $0.4 \mathrm{MPa}$, the force was found to be $18 \mathrm{~N}$. The efficiency is thus $\frac{18}{36}=50 \%$, because of losses due to friction in the sliding parts, and pressure drops due to small leakages.

\section{Needle holder}

The needle holder's central shaft consists of two parts that are connected by a bayonet mount and accomodates a 14gauge $(2.1 \mathrm{~mm})$ needle. At either end of the shaft, $\varnothing 18 \mathrm{~mm}$ three-way ball joints are installed. These joints consist of three parts, shown in Figure 4. The ball part is enclosed by two identical socket parts that are interlocked by a revolute joint inspired by the bayonet mount. Because each socket part features both a rim and a groove, these can revolute over more than $180^{\circ}$ in the interlocked state. Each socket part in turn is rigidly attached to the rack of the stepper motor. The joint connecting rack 5 to the needle holder is a revolute joint, using a $3 \mathrm{~mm}$ screw as the pin (rack 5 connects points $B^{\prime}$ and $B 5$ as shown in figure 5).

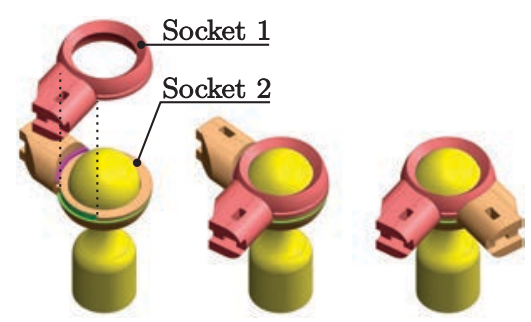

Fig. 4: Three-way ball joint mechanism, consisting of one ball part and two interlocked socket halves.

\section{KINEMATICS}

\section{A. Inverse kinematics}

To derive the inverse position kinematics of Stormram 2, it is represented as a skeleton with nine critical points, namely:
- Four points of the needle holder : $E$ (End-Effector), $A, B$ and $C$.

- Five points representing the centres of the five $45 \mathrm{~mm}$ ball joints located in the base : $B 1, B 2, B 3, B 4, B 5$.

The variables $L_{1} . . L_{5}$ represent the lengths of the five racks. These define the distances from the centres of the ball joints $B 1 . . B 5$ to the corresponding needle holder points $A, B$ and $C$.

Point $B$ is located on the line connecting points $E, A$ and $C$. The actual joint connecting the rack 5 is situated at a lower point $B^{\prime}$. Therefore, $L_{5}$ should be the length between $B^{\prime}$ and $B 5$, however, to simplify the geometry of the robot, $L_{5}$ is approximated to the distance between points $B$ and $B 5$. This difference is compensated for in the software implementation of the inverse position kinematics.

Figures 5 (a-d) illustrate the aforementioned points and variables in more detail. In figure 5(d), the Robot Coordinate System $\Psi^{R}$ uniquely determines the coordinates of the critical points. Furthermore, the five lengths $L_{1}, L_{2}, L_{3}, L_{4}$ and $L_{5}$ provide a desired end-effector $(E)$ position and orientation. Stormram 2 consists of five pneumatic linear stepper actuators and can therefore control the endeffector in five degrees of freedom, i.e. translation in $x, y, z$, rotation around $y$ (denoted by $\gamma$ ) followed by rotation around $x$ (denoted by $\alpha$ ) - as illustrated in figure 6 .

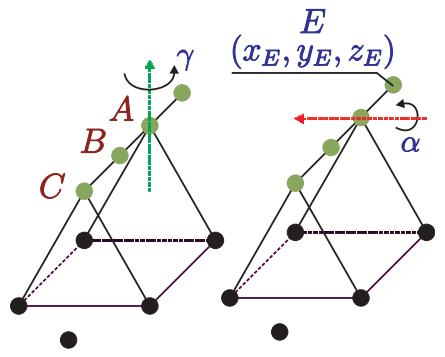

Fig. 6: End-effector $E$ Position and orientation.

The first step in determining the lengths $L_{1}, L_{2}, \ldots L_{5}$ from the given desired end-effector position $x_{E}, y_{E}, z_{E}$ and orientation $\gamma, \alpha$, is to obtain the relations between the needle

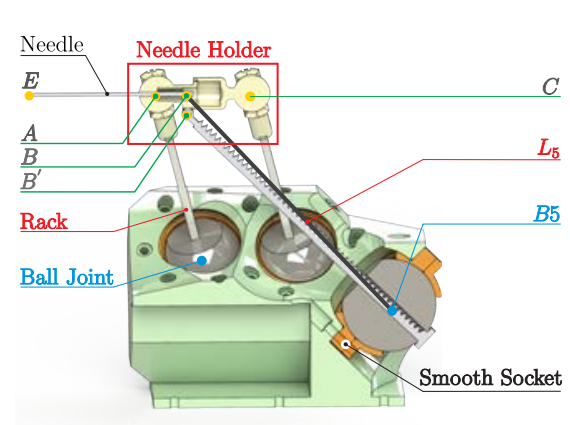

Sectional Side View

(a)

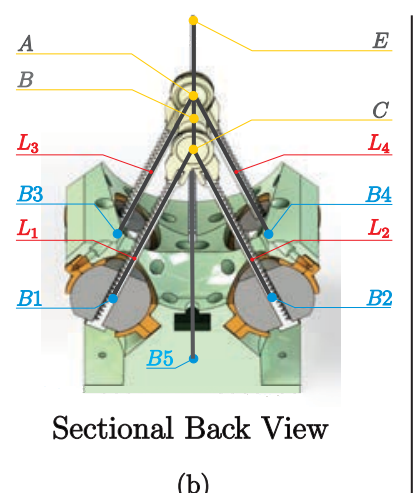

(b)

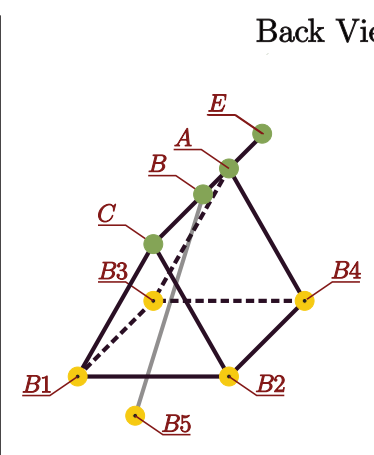

(c)

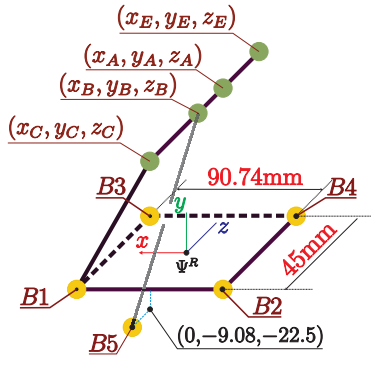

(d)

Fig. 5: (a)-(b) Labeled SOLIDWORKS assembly of Stormram 2 showing all the critical points of the needle holder, endeffector $\left(A, B, B^{\prime}, C\right.$ and $\left.E\right)$, the centre of the ball joints $(B 1, B 2, B 3, B 4$ and $B 5)$ and the connections between the points in different views. (c) Labeled sketch of Stormram 2's back view showing all the critical points (d) Labeled sketch of Stormram 2's back view showing the Robot Coordinate System $\left(\Psi^{R}\right)$, the coordinates of all points and other relevant dimensions. 
holder's critical points $(A, B$ and $C$ ) and the end-effector point $E$. The relation between point $A$ and $E$ is given by:

$$
\begin{aligned}
& x_{A}=x_{E}-L_{A E} \sin (\gamma) \\
& y_{A}=y_{E}+L_{A E} \cos (\gamma) \sin (\alpha) \\
& z_{A}=z_{E}-L_{A E} \cos (\gamma) \cos (\alpha)
\end{aligned}
$$

where $\left(x_{A}, y_{A}, z_{A}\right)$ is the position of point $A$ and $L_{A E}$ is the length between points $A$ and $E$ with a value $62.43 \mathrm{~mm}$. Similarly, the relation between point $A$ and $C$ is given by:

$$
\begin{aligned}
& x_{C}=x_{A}-L_{A C} \sin (\gamma) \\
& y_{C}=y_{A}+L_{A C} \cos (\gamma) \sin (\alpha) \\
& z_{C}=z_{A}-L_{A C} \cos (\gamma) \cos (\alpha)
\end{aligned}
$$

where $\left(x_{C}, y_{C}, z_{C}\right)$ is the position of point $C$ and $L_{A C}$ is the length between points $A$ and $C$ with a value $45.83 \mathrm{~mm}$. Next, the position of point B is derived. From figure 5 and 6 , point $\mathrm{B}$ lies between points $A$ and $C$. In general the position of a point (in this case $B$ ) on a line segment $(A C)$ is given by:

$$
\begin{aligned}
x_{B} & =\lambda x_{C}+(1-\lambda) x_{A} \\
y_{B} & =\lambda y_{C}+(1-\lambda) y_{A} \\
z_{B} & =\lambda z_{C}+(1-\lambda) z_{A}
\end{aligned}
$$

where $\lambda$ is the ratio of the known length between points $A$ and $B$ (i.e. $L_{A B}$ ) with a value $15.07 \mathrm{~mm}$ to the known length $L_{A C}$.

$$
\lambda=\frac{L_{A B}}{L_{A C}}=\frac{15.07}{45.833}=0.3288
$$

With the position of points $A, B$ and $C$ defined and evaluated, the lengths between the aforementioned points and the known ball joint centers $(B 1, B 2, B 3, B 4, B 5)$ are defined using the distance between two points mathematical relation:

$$
\left(x_{m}-x_{n}\right)^{2}+\left(y_{m}-y_{n}\right)^{2}+\left(z_{m}-z_{n}\right)^{2}=\left(L_{k}\right)^{2}
$$
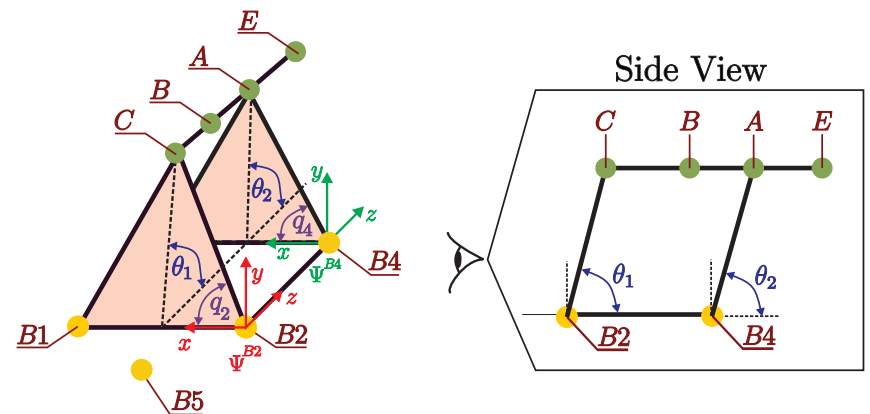

Fig. 7: Labeled sketch of Stormram 2's back view showing all the critical points, two newly added parameters $q_{1}$ and $q_{2}$ and the two intermediate coordinate systems $\Psi^{B 2}$ and $\Psi^{B 4}$. where $m=A, B, C, n=B 1 \ldots B 5$ and $k=1 \ldots 5$. The final step is to solve for the lengths $L_{1}, \ldots L_{5}$, given a desired $x_{E}, y_{E}, z_{E}, \gamma, \alpha$.

\section{B. Forward kinematics}

In this section, the forward position kinematics of Stormram 2 is derived. The basic idea here is to determine the end-effector position based on given lengths $L_{1}, L_{2}, L_{3}, L_{4}$ and $L_{5}$. In order to obtain the forward kinematic relations, a direct approach would be to utilise the equations obtained in the previous section (Inverse Position Kinematics) and reformulate them to obtain 5 equations in terms of the end-effector Cartesian position and orientation $\left(x_{E}, y_{E}, z_{E}, \gamma, \alpha\right)$. This could be done by substituting equations (1) to (9) into equation (11) resulting in five highly non-linear simultaneous equations.

Solving the five highly non-linear equations is computationally expensive and therefore a more efficient approach is required. This is achieved by introducing two new parameters $\theta_{1}$ and $\theta_{2}$ into Stormram 2's kinematic relations and solving 2 equations instead of the 5 equations. Figure 7 illustrates the location of both parameters. In addition, two intermediate parameters $q_{1}$ and $q_{2}$ and two intermediate coordinate systems $\Psi^{B 2}$ and $\Psi^{B 4}$ are defined in order to obtain the final equations.

To begin with, using the cosine rule $q_{2}$ and $q_{4}$ are obtained as follows:

$$
\begin{aligned}
& q_{2}=\arccos \left(\frac{\left(L_{2}\right)^{2}+90.748^{2}-\left(L_{1}\right)^{2}}{2 \cdot L_{2} \cdot 90.748}\right) \\
& q_{4}=\arccos \left(\frac{\left(L_{4}\right)^{2}+90.748^{2}-\left(L_{3}\right)^{2}}{2 \cdot L_{4} \cdot 90.748}\right)
\end{aligned}
$$

Next, the position of critical point $A$ is obtained with respect to the coordinate system $\Psi^{B 4}$ and the position of critical point $C$ is obtained with respect to the coordinate system $\Psi^{B 2}$ :

$$
\begin{array}{ll}
x_{A}^{B 4}=L_{4} \cos \left(q_{4}\right) & x_{C}^{B 2}=L_{2} \cos \left(q_{2}\right) \\
y_{A}^{B 4}=L_{4} \sin \left(q_{4}\right) \sin \left(\theta_{2}\right) & y_{C}^{B 2}=L_{2} \sin \left(q_{2}\right) \sin \left(\theta_{1}\right) \\
z_{A}^{B 4}=L_{4} \sin \left(q_{4}\right) \cos \left(\theta_{2}\right) & z_{C}^{B 2}=L_{2} \sin \left(q_{2}\right) \cos \left(\theta_{1}\right)
\end{array}
$$

To obtain the position vectors with respect to $\Psi^{R}$, Homogeneous matrices are utilised. The general form of the $4 \times 4$ homogeneous matrix is given by:

$$
H_{i}^{j}=\left[\begin{array}{cc}
R_{i}^{j} & o_{i}^{j} \\
\mathbf{0}^{T} & 1
\end{array}\right] \quad o_{i}^{j}=\left[\begin{array}{lll}
o_{x_{i}}^{j} & o_{y_{i}}^{j} & o_{z_{i}}^{j}
\end{array}\right]^{T}
$$

where $R_{i}^{j}$ represents the $3 \times 3$ rotation matrix from coordinate system $i$ to coordinate system $j, o_{i}^{j}$ represents the $3 \times 1$ offset between the origins of the coordinate systems $i$ and $j$. The positions of points $A$ and $C$ are transformed as follows: 


$$
\begin{aligned}
& p_{A}^{R}=H_{B 4}^{R} \cdot p_{A}^{B 4} \\
& p_{C}^{R}=H_{B 2}^{R} \cdot p_{C}^{B 2}
\end{aligned}
$$

where $p_{A}^{B 4}, p_{C}^{B 2}$ are the position vectors of points $A$ and $C$ with respect to the coordinate systems $\Psi^{B 4}$ and $\Psi^{B 2}$ respectively, $p_{A}^{R}, p_{C}^{R}$ are the position vectors with respect to the coordinate system $\Psi^{R}$ and $H_{B 4}^{R}, H_{B 2}^{R}$ are the homogeneous matrix transformations from coordinate systems $\Psi^{B 4}$ to $\Psi^{R}$ and $\Psi^{B 2}$ to $\Psi^{R}$ respectively.

$$
H_{B 4}^{R}=\left[\begin{array}{cc}
\mathbf{I} & o_{B 4}^{R} \\
\mathbf{0}^{T} & 1
\end{array}\right], \quad H_{B 2}^{R}=\left[\begin{array}{cc}
\mathbf{I} & o_{B 2}^{R} \\
\mathbf{0}^{T} & 1
\end{array}\right]
$$

In both cases, the rotation matrices $R_{B 2}^{R}$ and $R_{B 4}^{R}$ are identity matrices. This is because, the $x, y, z$ axes of all three coordinate systems are oriented in the same direction. The offset $o_{B 2}^{R}$ is the origin's position of coordinate system $\Psi^{B 2}$ with respect to the coordinate system $\Psi^{R}$ (the same applies for $o_{B 4}^{R}$ ). Furthermore, to satisfy the dimensions of the homogeneous matrix, the position vectors of critical points are written in the following manner:

$$
p_{v}^{l}=\left[\begin{array}{llll}
x_{v}^{l} & y_{v}^{l} & z_{v}^{l} & 1
\end{array}\right]
$$

where $p_{v}^{l}$ is arbitrary point $v$ defined with respect to coordinate system $l$. With the positions of points $A$ and $C$ defined with respect to the same coordinate system $\Psi^{R}$, the position of point $B$ is obtained in a similar manner to Equations (7) to $(9)$

$$
\begin{aligned}
x_{B}^{R} & =\lambda x_{C}^{R}+(1-\lambda) x_{A}^{R} \\
y_{B}^{R} & =\lambda y_{C}^{R}+(1-\lambda) y_{A}^{R} \\
z_{B}^{R} & =\lambda z_{C}^{R}+(1-\lambda) z_{A}^{R}
\end{aligned} \quad p_{B}^{R}=\left[\begin{array}{llll}
x_{B} & y_{B} & z_{B} & 1
\end{array}\right]^{T}
$$

In order to evaluate the forward kinematics, the following two equations are formulated: (1) The distance between points $p_{A}^{R}$ and $p_{C}^{R}$ and (2) The distance between points $p_{B}^{R}$ and $p_{B 5}^{R}$. For (1):

$$
\left|p_{A}^{R}-p_{C}^{R}\right|=L_{A C}
$$

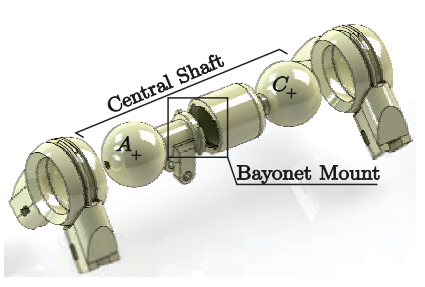

(a)

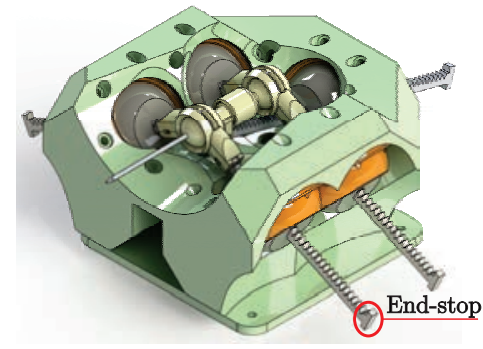

(b)

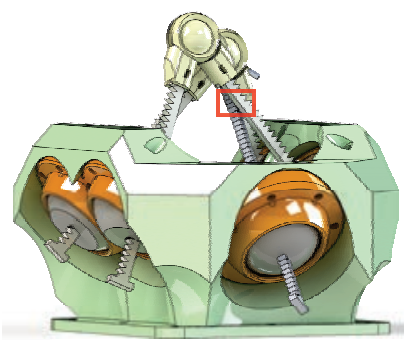

(c)

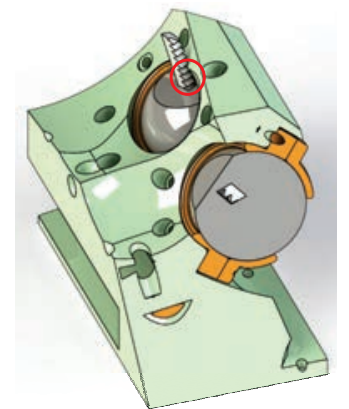

(d)

Fig. 8: (a) Disassembled needle holder, (b) Stormram 2's racks' end-stops, Collisions between (c) racks (d) rack and the base. 


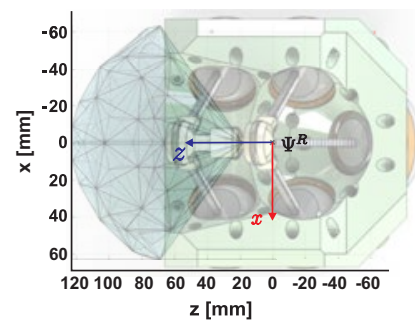

(a)

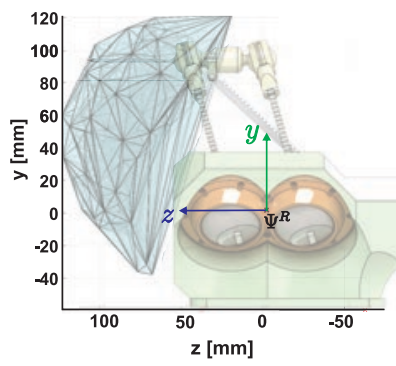

(b)

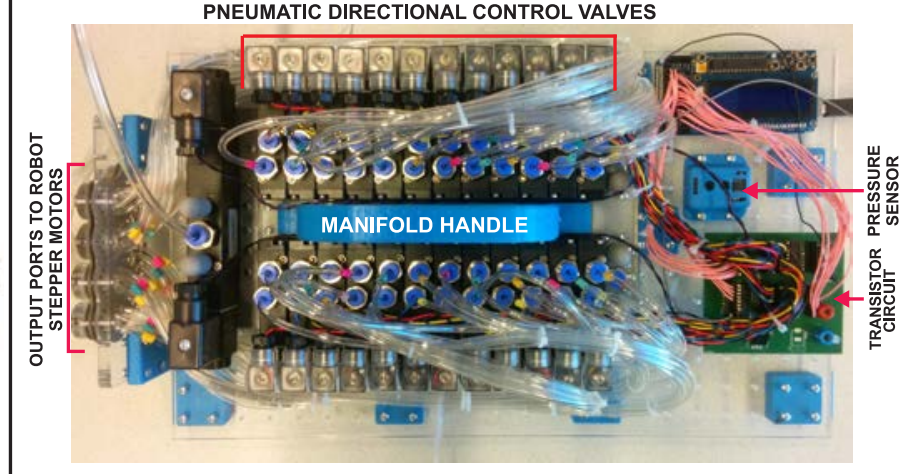

(c)

Fig. 10: (a) Top view (b) Side view of Stormram 2's reachable workspace, (c) Computerized valve manifold.

The collisions appear between the following components: (1) Needle holder parts (sockets and central shaft - see figure 8(a)), (2) Racks' (interconnected links) end-stops and the ball joints (see figure 8(b) for the end-stops), (3) Racks (see figure 8 (c)), (4) Racks and the base of the robot (see figure 8(d)) and (5) Racks and the needle holder.

Figure 9 illustrates the kinematic constraint due to the restriction in the relative configuration between the sockets of the needle holder.

The angle defined in the figure above $: \phi_{C}$ is computed using the cosine rule, as shown below:

$$
\phi_{C}=\arccos \left(\frac{\left(L_{1}\right)^{2}+\left(L_{2}\right)^{2}-90.74^{2}}{2 \cdot L_{1} \cdot L_{2}}\right)
$$

Based on the design of Stormram 2, the minimum value of $\phi_{C}$ is $50^{\circ}$, i.e. $\phi_{C \min }=50^{\circ}$. A similar analysis is carried out on the remaining kinematic constraints and mathematical expressions (similar to 20) are obtained. Taking into account the kinematic constraint analysis and the forward position kinematics algorithm, the reachable space of the needle tip (point $E$ ) is investigated. The reachable workspace, defined as the set of reachable positions of point $E$, is a $3 \mathrm{D}$ pointcloud which is visualized in figure 10 (a) and (b).

\section{Measurements}

\section{A. Experimental setup}

Figure 13 illustrates an overview of the experimental setup. To conduct experiments, a breast phantom made of PVC with plasticizer, filled with fish oil capsules, was immobilized in a frame and placed in the MRI scanner (see figure 13). When scanning using the 3D Balanced Gradient Echo sequence in the 0.25T Esaote G-scan Brio MRI scanner, good contrast is visible between the phantom and fish oil capsules (see figure 12).

As illustrated in figure 13, the MRI-compatible robot (Stormram 2), equipped with four markers at known positions, is positioned next to the breast phantom. In order to drive the pneumatic stepper motors with appropiate waveforms, a computerized valve manifold (see figure 10 (c)) is developed to operate Stormram 2 and guide the biopsy needle. The manifold allows the user to operate the robot at a distance of up to 7 metres, which is required because the controller cannot be placed inside the MRI room itself. Consequently, transmission delays in the pneumatic lines restrict the motor's stepping frequency to approximately 5 $\mathrm{Hz}$, depending on the valves types and tube dimensions. The manifold is computer-controlled and operated by a graphical user interface designed and running in MATLAB.

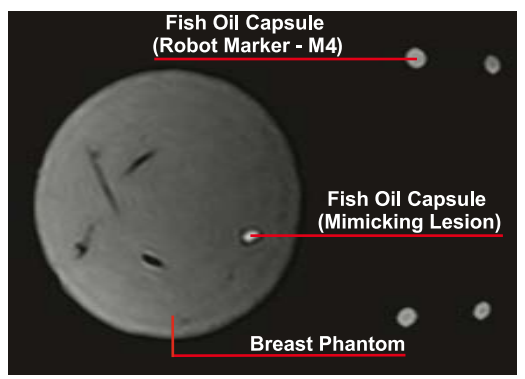

Fig. 12: Labeled MRI image of Stormram 2 and the breast phantom.

\section{B. Method}

The experiments are conducted on two breast phantoms with different stiffnesses and lesions of different sizes, to emulate the physical differences between patients. In the stiff breast phantom (made of $100 \%$ ( $600 \mathrm{~g}$ ) plastileurre), two $15 \mathrm{~mm}$-sized fish oil capsules are targeted. In the soft breast phantom (made of 85\% (510 g) of plastileurre and 15\% (90 g) of assouplissant plastileurre), one fish oil capsule sized $7 \mathrm{~mm}$ is targeted.

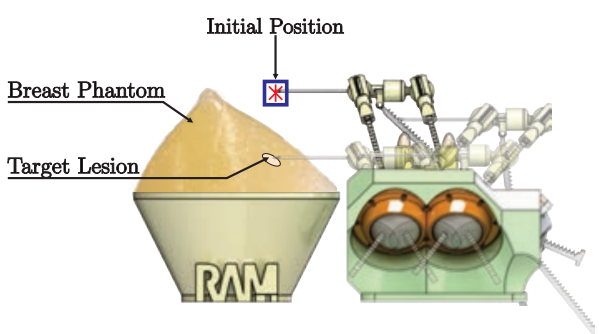

Fig. 12: Robot and needle tip movement.

The following steps are taken: (1) localize lesion using MRI (pre-targeting scan), (2) compute desired end-effector 

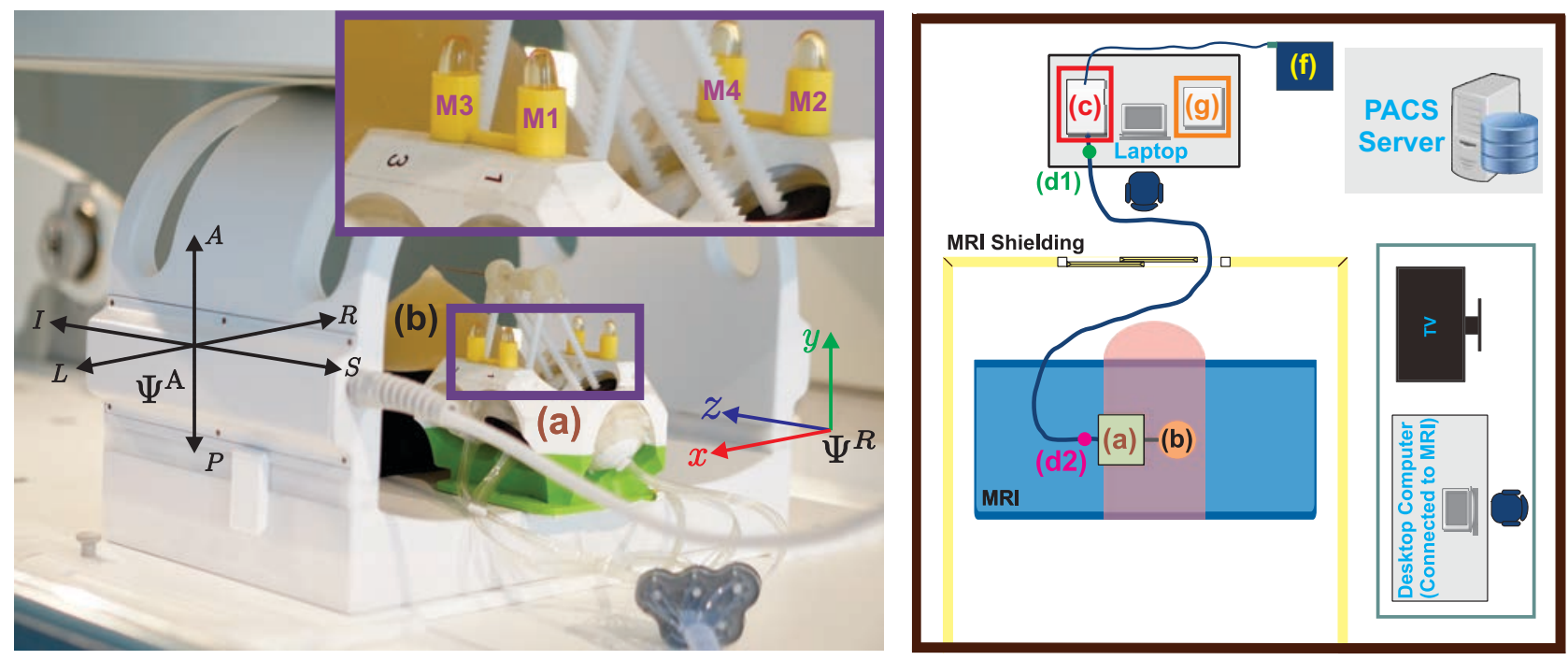

Fig. 13: (a) MRI-compatible robotic breast biopsy system (Stormram 2) with 4 markers (M1 ... M4), (b) Breast phantom with markers, (c) Solenoid valves pneumatic distributor which is also referred to as the computerized valve manifold (see figure 10(c)), (d1 and d2) Pneumatic tubes connecting the manifold to Stormram 2, (e) Portable Air Compressor, (f) MRI scanner - Esaote G-scan Brio 0.25T, (g) 24V/2.5A power supply. $\Psi^{A}$ is the anatomical coordinate system (also called patient coordinate system).

(needle) position $\left(x_{E}^{R}, y_{E}^{R}, z_{E}^{R}, \gamma, \alpha\right)$, (3) apply inverse kinematics algorithm and obtain desired lengths $L_{1} \ldots L_{5}$, (4) actuate the pneumatic linear stepper motors to achieve computed lengths (see figure 12), (5) take another MRI scan (post-targeting scan), (6) check if end-effector (needle tip) targeted the lesion, and perform accuracy analysis.

The efficiency of the system is also investigated, by recording the time taken for the computer-controlled MRI guided localization procedure.

\section{Results}

Figure (14) illustrates the pre- and post-targeting segmented scans of the first lesion in the stiff breast phantom. Different views are shown to illustrate what happens once the needle enters the breast phantom and targets the lesion (marker). It can be observed that the needle has pierced the capsule.
Figure 15 (left) shows one MRI slice. The target lesion is projected on it. A black gap can be seen in the vicinity of the target lesion coordinate. This gap measures approximately $8 \mathrm{~mm}$ in width, while the needle is only $2.1 \mathrm{~mm}$. The needle is projected on the MRI slice in the center of the gap, as we can assume that the artifact is symmetrical around the needle. The distance between the needle tip and the target lesion in all 3 axes can now be derived, which is a measure of the tip accuracy. There is also an uncertainty in the measured needle tip location. For each of the three targeted lesions, the $x, y$ and $z$ components of the distance between the measured needle tip position and target lesion, along with its uncertainties, are plotted.

The needle successfully targeted all three lesions with a resultant error $e_{r}=\sqrt{\left(e_{x}\right)^{2}+\left(e_{y}\right)^{2}+\left(e_{z}\right)^{2}}$ ranging from 4.7 and $7.3 \mathrm{~mm}$ with an uncertainty of approximately $2 \mathrm{~mm}$.

The time taken for the procedure was measured to be

Stiff breast phantom Lesion 1 Pre-Targeting

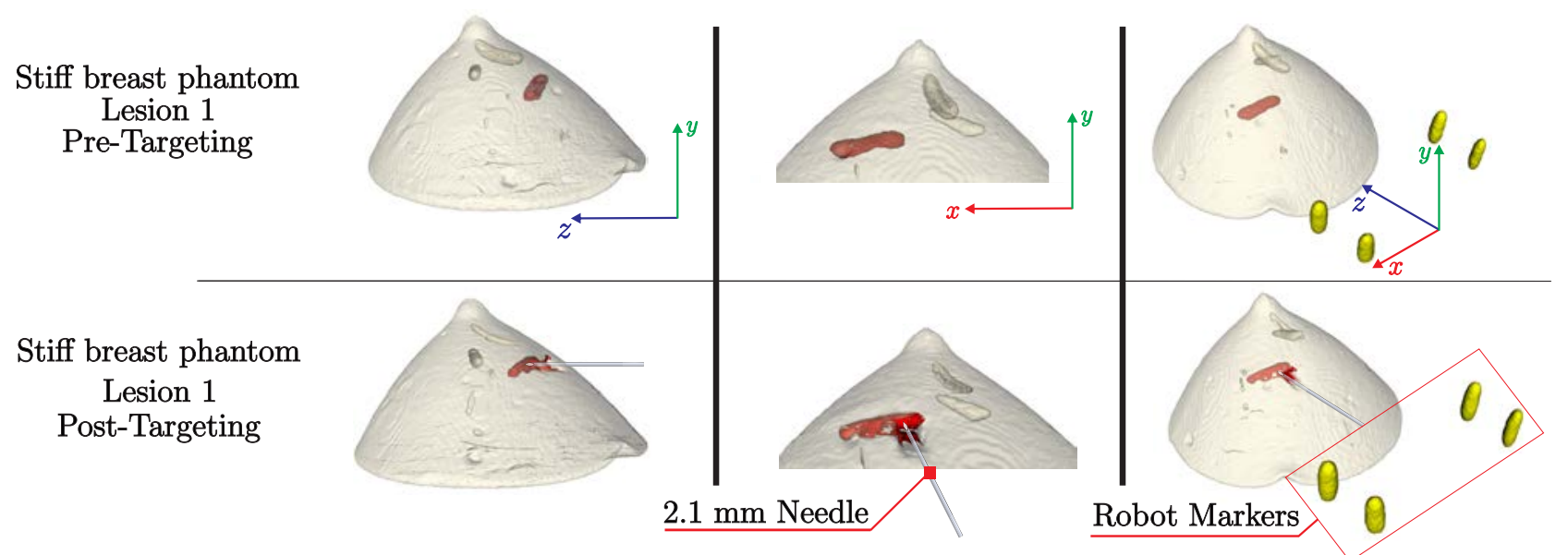

Fig. 14: Rendering using 3D SLICER of the stiff breast, targeting the lesion with a size $15 \mathrm{~mm}$. 


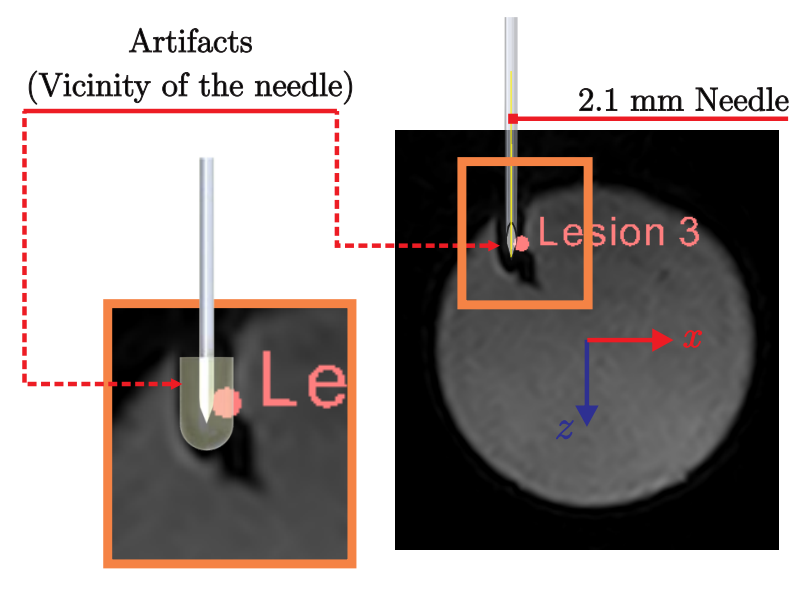

(a)

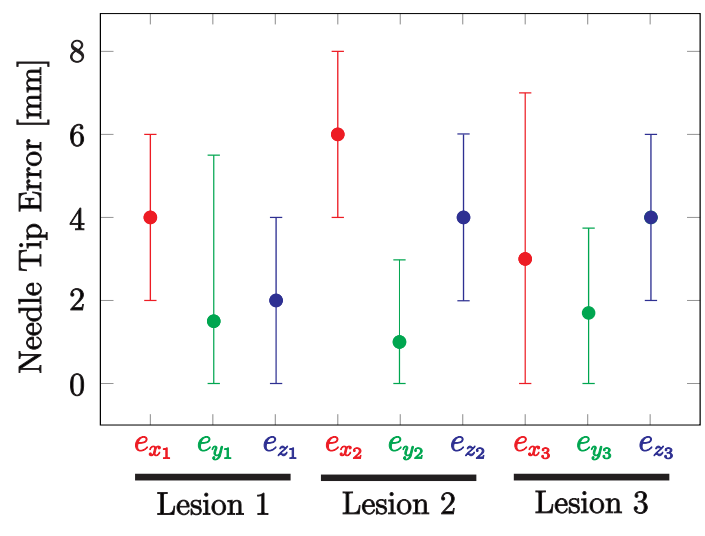

(b)

Fig. 15: (a) The artifacts (yellow) in the MRI scan caused by the titanium (metallic) needle, (b) Error bar plot.

approximately 31 minutes.

\section{Discussion}

Needle tip positioning errors of approximately $6 \mathrm{~mm}$ were measured. These errors are a result of:

- Possible breast tissue deformations.

- Approximation of length $L_{5}$ (see figure 5) and misalignment of points $B$ and $B^{\prime}$.

- Clearances in the ball joints adding up to $2 \mathrm{~mm}$ backlash in the needle tip.

- $\pm 1 \mathrm{~mm}$ calibration error in each axis with regards to the homing procedure, leading to approximately $2 \mathrm{~mm}$ needle tip positioning error.

- An average segmentation error of $1 \mathrm{~mm}$.

- Discretization of the racks because of the $1 \mathrm{~mm}$ step size, causing needle tip position errors ranging from $0.3 \mathrm{~mm}$ to $8.3 \mathrm{~mm}$, with an average error of $0.8 \mathrm{~mm}$. There are two possible ways to reduce this error, namely: (1) reducing the step size, and (2) sweeping over different combinations $L_{1} \ldots L_{5}$ in the neighbourhood of the computed lengths combination, in order to optimize the lengths values, minimize error and achieve sub-mm accuracy.

The time needed to take a good quality 3D Balanced Gradient Echo scan with the 0.25T scanner is approximately 5:30 minutes, plus 2:30 minutes post-processing. Taking two complete scans therefore takes 16 minutes. This can be reduced significantly by using a higher field strength MRI scanner (1.5T or more) or by optimizing the scanning sequence. The robot could also be operated at higher speed by using a different software architecture. This way, the 32 minutes could be reduced to 12 minutes.

\section{CONCLUSION}

An innovative, pneumatically actuated 5 DOF MRIcompatible breast biopsy system with computerized controller has been developed. It was able to target lesions in a breast phantom with an accuracy of approximately $6 \mathrm{~mm}$ within 31 minutes.
The developed system has the potential to improve accuracy and efficiency of current MRI-guided breast biopsy procedures, reducing patient discomfort and the number of false negative biopsies.

\section{A. Future improvements}

A good fixation system is necessary to avoid movements of the breast. Also, a biopsy gun firing mechanism needs to be built in the system in order to perform the actual biopsy. Improvements in accuracy are possible by redesigning the homing procedure and smaller stepper motor step sizes, while the procedure time can be reduced by using quicker MRI scans and a more efficient software architecture. Furthermore, the kinematic design could be improved to increase the workspace to the full volume of the breast.

\section{REFERENCES}

[1] Hauth, E. A., et al., "MR-guided vacuum-assisted breast biopsy with a handheld biopsy system: clinical experience and results in postinterventional MR mammography after 24 h," European radiology, vol.18, no.1, pp.168-176, 2008.

[2] N. Hungr, I. Bricault, P. Cinquin and C. Fouard, "Design and Validation of a CT- and MRI-Guided Robot for Percutaneous Needle Procedures," IEEE Trans. Robot., vol.32, no.4, pp.973-987, Aug. 2016.

[3] H. Su, et al., "Piezoelectrically Actuated Robotic System for MRIGuided Prostate Percutaneous Therapy," IEEE/ASME Trans. Mechatronics, vol.20, no.4, pp.1920-1932, Aug. 2015.

[4] B. Yang, S. Roys, U-X. Tan, M. Philip, H. Richard, R.P. Gullapalli and J.P. Desai, "Design, development, and evaluation of a master-slave surgical system for breast biopsy under continuous MRI," Int. J. Robot. Res., vol. 33, no. 4, pp. 616-630., 2014.

[5] M. Li, D. Mazilu, A. Kapoor and K. A. Horvath, "MRI Compatible Robot Systems for Medical Intervention," Advances in Robot Manipulators, Ed. Ernest Hall., April 2010, pp. 443-458.

[6] J. A. Cunha, et al., "Toward adaptive stereotactic robotic brachytherapy for prostate cancer: Demonstration of an adaptive work flow incorporating inverse planning and an MR stealth robot," Minimally invasive therapy \& allied technologies : MITAT : official journal of the Society for Minimally Invasive Therapy, vol. 19, no. 4, pp. 189-202, 2010.

[7] V. Groenhuis and S. Stramigioli, "Laser-Cutting Pneumatics," IEEE/ASME Trans. Mechatronics, vol. 21, no. 3, pp. 1604-1611, June 2016.

[8] V. Groenhuis, J. Veltman and S. Stramigioli, "Stormram 2: A MRIcompatible pneumatic robotic system for breast biopsy," Proc. of The Hamlyn Symp. on Medical Robotics, pp. 52-53, June 2016. 